\title{
纳米金颗粒增强信号的表面等离子体共振生物传感器用于甲氧檗因 高灵敏检测的研究
}

\author{
王青 朱红志羊小海* 王柯敏* 杨丽娟 丁静 \\ (湖南大学化学生物传感与计量学国家重点实验室 化学化工学院 生物纳米与分子工程湖南省重点实验室 \\ 长沙 410082)
}

\begin{abstract}
摘要 报道了一种基于表面等离子体共振(SPR)生物传感器的高灵敏检测抗癌药物甲氧檗因的新方法. 分别在纳米金 颗粒和金膜表面修饰富含腺嘌呤(A)的 DNA 链，当存在甲氧檗因时，由于一个甲氧檗因分子可与 4 个 $\mathrm{A}$ 碱基相结合，从 而使得修饰在纳米金颗粒和金膜表面的 DNA 形成稳定的双链结构, 进而将功能化纳米金颗粒捕获在金膜表面. 由于纳 米金颗粒与金膜之间的电场耦合效应可增强 SPR 信号，从而可实现对小分子甲氧檗因的高灵敏、特异性检测. 本方法 的检测下限低至 $0.07 \mathrm{pmol} / \mathrm{L}$ ，相对比色法和荧光法而言，降低了约 $5 \sim 6$ 个数量级. 以 4 种药物(盐酸小檗碱、青霉素 G、 硫酸庆大霉素、5-氟尿嘧啶)作为对照考察了该传感器的选择性, 结果表明该传感器具有较好的选择性.
\end{abstract}

关键词＼cjkstart表面等离子体共振; 纳米金颗粒; 甲氧檗因; DNA; 生物传感器

\section{High Sensitive Coralyne Detection by Using of Au Nanoparticles-Enhanced Surface Plasmon Resonance Biosensor}

\author{
Wang, Qing Zhu, Hongzhi Yang, Xiaohai* Wang, Kemin* \\ Yang, Lijuan Ding, Jing
}

(State Key Laboratory of Chemo/Biosensing and Chemometrics, College of Chemistry and Chemical Engineering, Key Laboratory for Bio-Nanotechnology and Molecular Engineering of Hunan Province, Hunan University, Changsha 410082)

\begin{abstract}
Coralyne, a kind of planar alkaloid, has more pronounced antitumor activity compared to other protoberberine alkaloids. It was found that coralyne could induce the adenine (A)-rich DNA oligonucleotides to form a double strand DNA, with a stoichiometry of one coralyne per four adenine bases. In this paper, a high sensitive approach for the detection of anticancer drug coralyne based on surface plasmon resonance (SPR) and the high selectivity and sensitivity recognition process of coralyne with the A-rich DNA oligonucleotides was established. Generally, it was difficult to detect small molecules directly using conventional SPR biosensors since the changes of refractive index, which was resulted by binding small molecules, were usually small. Here, the Au nanoparticles was introduced for enhancing the sensitivity. The A-rich DNA strands were modified on Au nanoparticles and on Au film respectively. In the presence of coralyne and the functional Au nanoparticles, the A-rich DNA strands could form the duplex through the specific coordination between coralyne and adenine, and then the functional Au nanoparticles were captured on the Au film. SPR signal was enhanced by the electronic coupling between the localized plasmon of Au nanoparticles and the surface plasmon wave associated with Au film. If only coralyne or the functional Au nanoparticles was present, the resonance wavelength was almost unchanged. It meant that there was no A-coralyne-A complex formed. Thus, this method could be used to detect coralyne, and the background was low. The results showed that the SPR sensor was highly sensitive, and the limit of detection was $c a .0 .07 \mathrm{pmol} / \mathrm{L}(S / N=3)$. It was about $5-6$ orders of magnitude lower than those of fluorescent and colorimetric methods. Moreover, four kinds of small molecule drugs (berberine hydrochloride, penicillin, gentamicin sulfate, and 5-fluorouracil) were used as control to investigate the selectivity of this SPR biosensor. For penicillin, gentamicin sulfate, and 5-fluorouracil, all of the resonance wavelength shifts were lower than $0.4 \mathrm{~nm}$. For high concentration of berberine hydrochloride, the resonance wavelength shifts were $c a$. $0.5-0.6 \mathrm{~nm}$. The results showed that this SPR biosensor has good selectivity. This work may extend the application of SPR biosensors in drug discovery and development.
\end{abstract}

Keywords surface plasmon resonance; Au nanoparticles; coralyne; DNA; biosensor

\footnotetext{
*E-mail: yangxiaohai@hnu.edu.cn; kmwang@hnu.edu.cn

Received April 10, 2012; published May 26, 2012.

Project supported by the National Natural Science Foundation of China (Nos. 21190044, 21175035), National Basic Research Program (No. 2011CB911002), International Science \& Technology Cooperation Program of China (No. 2010DFB30300), and Program for New Century Excellent Talents in University (No. NCET-09-0338), Hunan Province Ordinary College Young Teachers Training Project (2009).

项目受国家自然科学基金(Nos. 21190044, 21175035)、973 计划(No. 2011CB911002)、科技部国际合作重大项目(No. 2010DFB30300)、教育部 “新世 纪优秀人才支持计划” (No. NCET-09-0338)和湖南省高等学校青年骨干教师培养对象基金(2009 年度)资助.
} 


\section{1 引言}

甲氧檗因是一种新月形的平面生物碱, 当与富含 $\mathrm{A}$ 碱基的 DNA 作用时, 一个甲氧檗因分子可特异性结合 四个 $\mathrm{A}$ 碱基 ${ }^{[1]}$. Wilson 等 ${ }^{[2]}$ 发现甲氧檗因对小鼠体内的 P388 和 L1210 白血病细胞有治疗作用, 并且与其它原小 檗碱类生物碱相比, 低毒性的甲氧檗因具有更高的抗肿 瘤活性. 目前, 基于甲氧檗因与富含 A 碱基的 DNA 的 特异性结合, 已经建立了多种用于甲氧檗因的检测方 法. 例如 $\mathrm{Lv}$ 等 ${ }^{[1]}$ 利用纳米金颗粒的免标记比色方法对 甲氧檗因进行快速检测, 检测下限为 $91 \mathrm{nmol} / \mathrm{L}$; 基于 类似的原理, $\mathrm{Xu}$ 等 ${ }^{[3]}$ 设计了基于纳米银颗粒的免标记比 色方法检测甲氧檗因, 其检测下限为 $1 \mu \mathrm{mol} / \mathrm{L} ; \mathrm{Lin}^{\text {等 }}{ }^{[4]}$ 利用荧光方法实现了对甲氧檗因检测, 检测限为 65 $\mathrm{nmol} / \mathrm{L}$; Wang 等 ${ }^{[5]}$ 报道了一种双偏振干涉检测方法, 通 过表面质量、厚度、折射率的变化实现对甲氧檗因的定 量和定性分析, 检测限均为 $\mu \mathrm{mol} / \mathrm{L}$ 级. 如能进一步提高 检测灵敏度, 对于检测微量生物样品中的甲氧檗因, 以 及研究该药物与核酸的相互作用具有重要价值.

表面等离子体共振技术(surface plasmon resonance, SPR)由于具有样品用量少、灵敏度高以及适于实时检测 等特点, 已广泛应用于蛋白质 ${ }^{[6,7]}$ 、核酸 ${ }^{[8]}$ 、小分子 ${ }^{[9 \sim 11]}$ 和离子[12]等目标物的检测. 通常, 对于小分子而言, 由 于其结合所引起的 SPR 信号非常小, 用常规的 SPR 传感 器难以直接检测. 鉴于纳米金颗粒的局域表面等离子体 和金膜表面等离子体波之间的电场耦合效应可以增强 SPR 信号 ${ }^{[13]}$, 据此构建的纳米金颗粒增强信号的 SPR 传 感器实现了对可卡因 ${ }^{[10]}$ 、腺苷 ${ }^{[1]}$ 等小分子的高灵敏检 测.

本文基于纳米金颗粒增强信号的 SPR 生物传感器 建立了高灵敏检测抗癌药物甲氧檗因的新方法. 同时选 取盐酸小檗碱、青霉素 $\mathrm{G}$ 、硫酸庆大霉素和 5-氟尿嘧啶 等四种小分子药物考察了该传感器的选择性.

\section{2 结果与讨论}

\section{1 检测原理}

检测原理如图 1 所示. 先将富含 $\mathrm{A}$ 碱基的巯基化 DNA 分别修饰在金膜表面和纳米金颗粒上, 当甲氧檗 因存在时, 通过其与 $\mathrm{A}$ 碱基的特异性结合, 将 DNA-AuNPs 捕获到 SPR 传感芯片表面, 可引起明显的 共振波长的移动; 当没有甲氧檗因存在的情况下, DNA-AuNPs 不能被捕获到 SPR 传感芯片表面, 则不发 生共振波长移动. 根据共振波长位移大小, 可实现对甲 氧檗因的检测.

\section{2 颈基化 DNA 修饰的纳米金颗粒的表征}

利用紫外-可见光谱表征了颈基化 DNA 修饰前后
纳米金颗粒光谱变化(图 2). 结果表明, 当纳米金颗粒表 面修饰颈基化 DNA 之后, 其最大吸收峰从 $518.0 \mathrm{~nm}$ 红 移至 $524.0 \mathrm{~nm}$, 这说明颈基化 DNA 已修饰到纳米金颗 粒表面. 根据 DNA-AuNPs 复合物的紫外-可见光谱中 最大吸收值 ${ }^{[14]}$, 可以计算出 DNA-AuNPs 复合物的浓度 为 $3.0 \mathrm{nmol} / \mathrm{L}$.

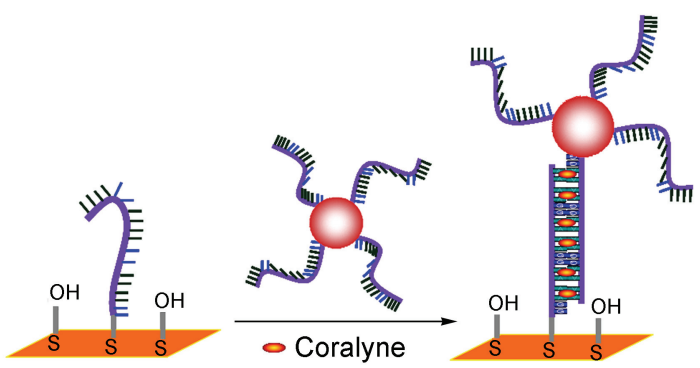

图 1 基于纳米金颗粒增强信号的 SPR 生物传感器的甲氧檗因检测示 意图

Figure 1 Scheme of coralyne detection based on Au nanoparticles enhanced SPR biosensor

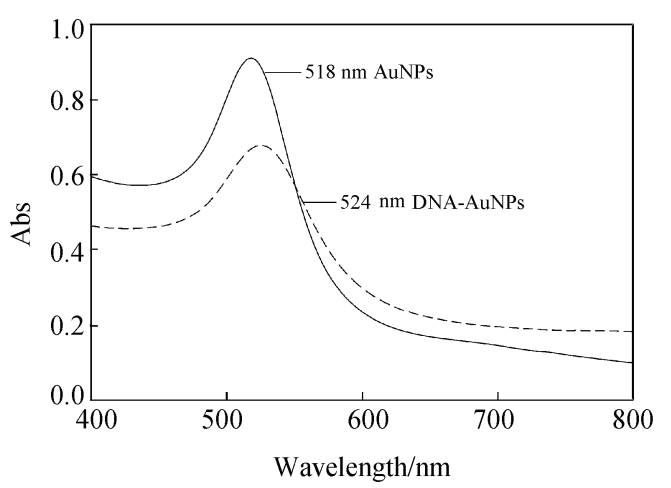

图 2 DNA 修饰前后纳米金颗粒紫外-可见光谱变化图

Figure 2 UV-vis spectra of AuNPs before and after modified DNA

\section{3 可行性考察}

参考文献 ${ }^{[15]}$, 采用计时电量法测定了金膜表面統 基化 DNA 探针的修饰密度. 结果表明, DNA 探针的修 饰密度为 $7.6 \times 10^{12}$ molecule $\cdot \mathrm{cm}^{-2}$. 这一数值与我们前 期工作的优化结果接近 ${ }^{[16]}$, 因此后续实验均采用此探 针修饰密度.

首先考察了该传感器检测甲氧檗因的可行性, 结果 如图 3 所示.

当注入甲氧檗因(终浓度为 $50 \mathrm{pmol} / \mathrm{L}$ ) 和 $\mathrm{DNA}$ AuNPs 的混合溶液时, 其 SPR 光谱从曲线 $\mathrm{a}$ 红移至曲线 $\mathrm{b}$, 共振波长移动 $2.2 \mathrm{~nm}$ (图 3A); 而仅注入 DNA-AuNPs 溶液或甲氧檗因溶液时, 其 SPR 光谱均基本保持不变 (图 3B、3C). 结果表明, 只有存在甲氧檗因时才能使 DNA-AuNPs 被捕获在金膜表面上, 以引起 SPR 光谱的 明显变化, 从而实现了甲氧檗因的检测. 

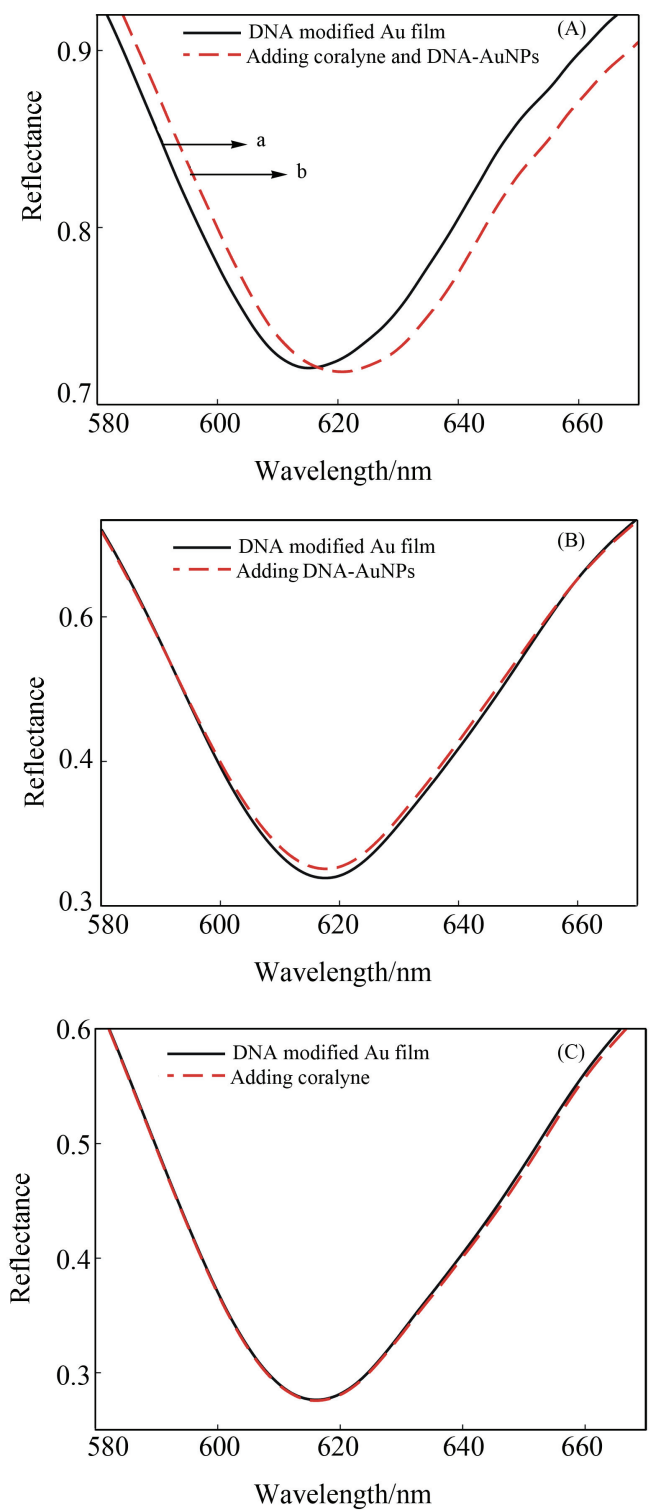

图 3 (A)加入 $50 \mathrm{pmol} / \mathrm{L}$ 甲氧檗因和 DNA 修饰的纳米金颗粒之后 SPR 光谱变化图; (B) 仅加入 DNA 修饰的纳米金颗粒之后 SPR 光谱变 化图; (C)仅加入 $50 \mathrm{pmol} / \mathrm{L}$ 甲氧檗因之后 $\mathrm{SPR}$ 光谱变化图

Figure 3 SPR spectra of DNA modified Au film after (A) adding 50 $\mathrm{pmol} / \mathrm{L}$ coralyne and DNA-AuNPs simultaneously; (B) only adding DNA-AuNPs; (C) only adding $50 \mathrm{pmol} / \mathrm{L}$ coralyne

\section{4 灵敏度的考察}

采用 SPR 生物传感器检测了不同浓度的甲氧檗因, 结果如图 4 所示. 结果表明, 随着甲氧檗因浓度的增加 (从 $0.1 \mathrm{pmol} / \mathrm{L}$ 至 $1.0 \mathrm{nmol} / \mathrm{L}$ ), SPR 共振波长位移也增加, 这主要是由于甲氧檗因浓度的增加使得被捕获到 SPR 传感芯片上的 DNA-AuNPs 数量增加所致. 以共振波长 位移 $(\Delta \lambda)$ 为纵坐标, 甲氧檗因浓度的对数值为横坐标作 图(见图 4 中小插图), 结果显示, 甲氧檗因浓度在 $1.0 \times$ $10^{-13} \sim 5.0 \times 10^{-11} \mathrm{~mol} / \mathrm{L}$ 之间时, 其浓度的对数与共振 波长位移呈线性相关, 线性回归方程为 $\Delta \lambda=0.6101 \mathrm{Log}$

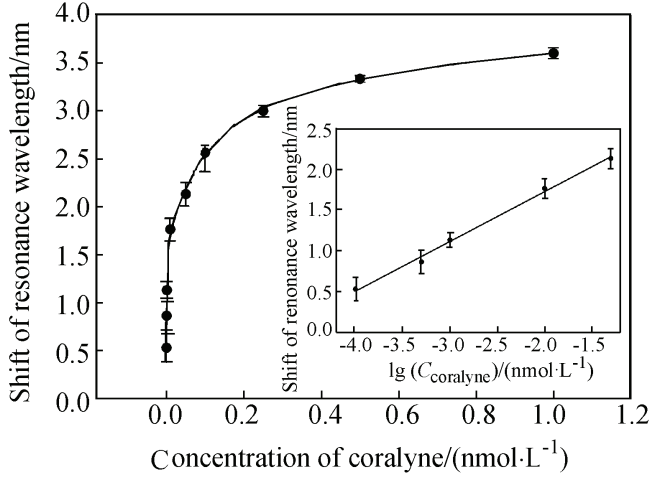

图 4 检测不同浓度的甲氧檗因. 小插图表示的是共振波长位移与甲 氧檗因浓度的对数呈线形关系

Figure 4 Detection of different concentrations of coralyne. The inset provides the linearity of resonance wavelength shift against the logarithm of coralyne concentrations

$C+2.9464$, 相关系数为 0.9978 . 该传感器的检测下限约 为 $0.07 \mathrm{pmol} / \mathrm{L}(S / N=3)$, 相对比色法 ${ }^{[1,3]}$ 和苂光法 ${ }^{[4,17]}$ 而 言, 降低了约 $5 \sim 6$ 个数量级.

\section{5 选择性的考察}

为考察该传感器的选择性，选取了盐酸小檗碱、青 霉素 G、硫酸庆大霉素、5-氟尿嘧啶四种小分子药物作 为对照. 结果如图 5 所示, 青霉素 G、硫酸庆大霉素和 5 -氟尿嘧啶这 3 种药物所引起的共振波长移动均小于 $0.4 \mathrm{~nm}$ ，说明对甲氧檗因的检测基本没有影响. 而较高 浓度的盐酸小檗碱可引起共振波长发生 $0.5 \sim 0.6 \mathrm{~nm}$ 的 移动，这可能是由于盐酸小檗碱具有与甲氧檗因相似的 结构, 对富含 A 碱基的核酸具有一定的结合能所致 ${ }^{[18]}$.

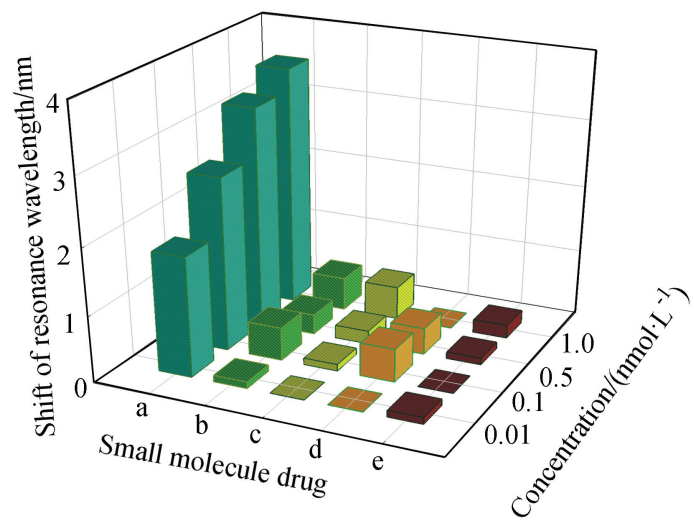

图 5 分别加入甲氧檗因和四种小分子药物所引起的共振波长移动. $\mathrm{a} \sim \mathrm{e}$ 分别为: 甲氧檗因, 盐酸小檗碱, 青霉素 $\mathrm{G}$, 硫酸庆大霉素和 5氟尿嘧啶

Figure 5 The resonance wavelength shift in the presence of coralyne and other four kinds of small molecule drugs. a-e: coralyne, berberine hydrochloride, penicillin, gentamicin sulfate, 5 -fluorouracil

\section{6 重现性的考察}

进一步考察了该传感器的重现性, 结果如图 6 所示, 对 $50 \mathrm{pmol} / \mathrm{L}$ 的甲氧檗因进行多次检测，可以看出前面 
4 次重复实验, 其共振波长位移为 $(2.1 \pm 0.1) \mathrm{nm}(n=4)$. 之后, 其共振波长位移降低, 说明该传感芯片经过 4 次 重复实验后性能降低. 同时发现, 该传感器的再生可以 利用 $10 \mathrm{mmol} / \mathrm{L} \mathrm{NaOH} / 0.1 \% \mathrm{SDS}$ 溶液进行.

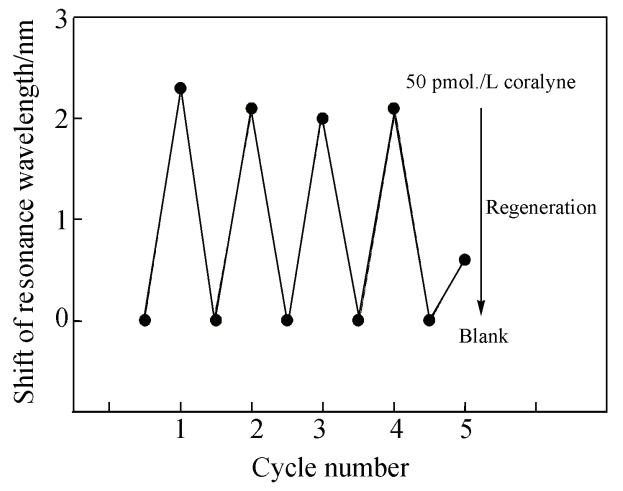

图 6 重现性考察, 传感芯片用 $10 \mathrm{mmol} / \mathrm{L} \mathrm{NaOH} / 0.1 \% \mathrm{SDS}$ 溶液进行 再生

Figure 6 Reproducibility of the resonance wavelength shift of sensor chip for blank and $50 \mathrm{pmol} / \mathrm{L}$ coralyne. The sensor chip was regenerated by $10 \mathrm{mmol} / \mathrm{L} \mathrm{NaOH} / 0.1 \% \mathrm{SDS}$

\section{3 结论}

基于小分子药物甲氧檗因对富含 A 碱基的 DNA 的 特异性识别能力, 利用纳米金颗粒作为信号放大技术, 构建了高灵敏检测甲氧檗因的 SPR 生物传感器, 检测下 限可低至 $0.07 \mathrm{pmol} / \mathrm{L}(S / N=3)$. 该 $\mathrm{SPR}$ 生物传感器的传 感芯片制备简便, 选择性高, 为小分子药物的检测和研 究小分子药物与核酸的相互作用提供了一种新方法.

\section{4 实验部分}

\section{1 试剂与仪器}

氯金酸(分析纯，国药集团化学试剂有限公司); 二 水合柠檬酸三钠(分析纯，上海试剂一厂); 放基己醇 $(\mathrm{MCH}, 97 \%$, 美国 Simga 公司); 三氯化六铵合钉(分析 纯, 百灵威科技有限公司); 甲氧檗因(分析纯, 百灵威 科技有限公司)、青霉素 G(分析纯, Amresco 公司)、硫酸 庆大霉素(分析纯, 合肥博美生物有限公司)、盐酸小檗 碱(分析纯，百灵威科技有限公司)、5-氟尿嘧啶(分析纯, 合肥博美生物有限公司). 药物均溶于 $0.01 \mathrm{~mol} / \mathrm{L} \mathrm{PBS}$ 缓 冲液 $(\mathrm{pH}=7$, 含 $0.3 \mathrm{~mol} / \mathrm{L} \mathrm{NaCl})$. 颈基化 DNA 探针购自 上海生工有限公司, 序列如下: 5'-HS- ${ }_{6} \mathrm{H}_{12}$-GGAAAAGGAAAAGGAAAA-3'. 如没有特别说明, 其余试剂均 为分析纯, 实验用水均为电阻率 $18.2 \mathrm{M} \Omega \cdot \mathrm{cm}$ 的纯净水.

本文中所用的基于波长调制的 SPR 传感器由本实 验室自行组装 ${ }^{[19]}$, 它主要由平行偏振光源、敏感元件、 分光系统、信号采集与处理系统等几部分构成. 仪器工 作时温度控制在 $25{ }^{\circ} \mathrm{C}$, 当共振波长位移 $(\Delta \lambda)$ 大于 0.4 $\mathrm{nm}$ 即可认为是信号.

\section{2 纳米金颗粒的制备、修饰与表征}

采用柠檬酸三钠还原方法制备纳米金颗粒，参照文 献 ${ }^{[16,20]}$ 将巯基化 DNA 修饰在纳米金颗粒表面，并采用 离心分离方法将 DNA-AuNPs 溶液中游离的单链 DNA 去除. 用紫外-可见分光光度计对纳米金颗粒修饰統基 化 DNA 前后的光谱变化进行表征.

\subsection{SPR 传感芯片的制作}

首先将清洗干净的金膜浸泡在 $100 \mu \mathrm{mol} / \mathrm{L}$ 颈基化 DNA 溶液中反应 $24 \mathrm{~h}$, 再用 $0.01 \mathrm{~mol} / \mathrm{L} \mathrm{PBS}$ 缓冲液反复 冲洗后用 $1 \mathrm{mmol} / \mathrm{L} \mathrm{MCH}$ 封闭处理 $1 \mathrm{~h}$, 最后用 0.01 $\mathrm{mol} / \mathrm{L}$ PBS 缓冲液反复冲洗, 即可制成 SPR 传感芯片.

\section{4 巯基化 DNA 在金膜表面修饰密度的测定}

金膜表面固定化 DNA 的修饰密度是通过计时电量 法测定的. 采用计时电量法测定分别有和无三氯化六铵 合钌的 $10 \mathrm{mmol} / \mathrm{L}$ Tris- $\mathrm{HCl}(\mathrm{pH}=7)$ 缓冲液中修饰了 DNA 的金膜表面电量. 根据参考文献 ${ }^{[15]}$ 可计算出金膜 表面 DNA 的修饰密度.

\section{5 甲氧檗因的检测}

首先在流通池中注入 $0.01 \mathrm{~mol} / \mathrm{L} \mathrm{PBS}$ 缓冲液, 记录 其 SPR 光谱; 然后分别加入由 $90 \mu \mathrm{L}$ DNA-AuNPs 溶液 和 $10 \mu \mathrm{L}$ 一系列不同浓度的甲氧檗因溶液组成的混合液 反应 $45 \mathrm{~min}$, 再用 $0.01 \mathrm{~mol} / \mathrm{L} \mathrm{PBS}$ 缓冲液反复冲洗, 记 录其 SPR 光谱. 采用 $10 \mathrm{mmol} / \mathrm{L} \mathrm{NaOH} / 0.1 \%$ 十二烷基磺 酸钠(SDS)溶液使 SPR 传感芯片再生.

选取盐酸小檗碱、青霉素 G、硫酸庆大霉素、5-氟 尿嘧啶 4 种药物, 考察该 SPR 生物传感器的选择性.

\section{References}

[1] Lv, Z. Z.; Wei, H.; Li, B. L.; Wang, E. K. Analyst 2009, 134, 1647.

[2] Wilson, W. D.; Gough, A. N.; Doyle, J. J.; Davidson, M. W. J. Med. Chem. 1976, 19, 1261.

[3] Xu, X.; Wang, J.; Yang, F.; Jiao, K.; Yang, X. Small 2009, 5, 2669.

[4] Lin, Y. H.; Tseng, W. L. Chem. Commun. 2011, 47, 11134.

[5] Wang, Y.; Wang, J.; Yang, F.; Yang, X. Anal. Chem. 2012, 84, 924.

[6] Homola, J. Chem. Rev. 2008, 108, 462.

[7] Situ, C.; Mooney, M. H.; Elliott, C. T.; Buijs, J. Trends Anal. Chem. 2010, 29, 1305.

[8] Altintas, Z.; Uludag, Y.; Gurbuz, Y.; Tothill, I. Anal. Chim. Acta 2012, 712, 138.

[9] Yang, X.-H.; Li, Z.-H.; Wang, Q.; Wang, K.-M. Acta Chim. Sinica 2007, 65, 1185 (in Chinese). (羊小海, 黎振华, 王青, 王柯敏, 化 学学报, 2007, 65, 1185.)

[10] Golub, E.; Pelossof, G.; Freeman, R.; Zhang, H.; Willner, I. Anal. Chem. 2009, 81, 9291.

[11] Wang, Q.; Huang, J. H.; Yang, X. H.; Wang, K. M.; He, L. L.; Li, X. P.; Xue, C. Y. Sens. Actuators B 2011, 156, 893.

[12] Wang, L.; Li, T.; Du, Y.; Chen, C.; Li, B.; Zhou, M.; Dong, S. Biosens. Bioelectron. 2010, 25, 2622

[13] Lyon, L. A.; Musick, M. D.; Smith, P. C.; Reiss, B. D.; Peña, D. J.; Natan, M. J. Sens. Actuators B 1999, 54, 118. 
[14] Kira, A.; Kim, H.; Yasuda, K. Langmuir 2009, 25, 1285.

[15] Adam, B. S.; Tonya, M. H.; Michael, J. T. Anal. Chem. 1998, 70, 4670.

[16] Wang, Q.; Yang, L. J.; Yang, X. H.; Wang, K. M.; He, L. L.; Zhu, J. Q. Anal. Chim. Acta 2011, 688, 163.

[17] Zhou, Y.-W.; Li, C.-M.; Xiong, Z.-H.; Huang, C.-Z. Acta Chim. Sinica 2012, 70, 352 (in Chinese). (周亚文, 李春梅, 熊祖洪, 黄承
志, 化学学报, 2012, 70, 352.)

[18] Giri, P.; Kumar, G. S. Arch. Biochem. Biophys. 2008, 474, 183.

[19] Yang, X. H.; Wang, Q.; Wang, K. M.; Tan, W. H.; Li, H. M. Biosens. Bioelectron. 2007, 22, 1106.

[20] Storhoff, J. J.; Elghanian, R.; Mucic, R. C.; Mirkin, C. A.; Letsinger, R. L. J. Am. Chem. Soc. 1998, 120, 1959. 


\section{Graphical Abstract}

\section{Article}

\section{High Sensitive Coralyne Detection by Using of Au Nanoparticles-Enhanced Surface Plasmon Resonance Bio- sensor}

Wang, Qing; Zhu, Hongzhi; Yang, Xiaohai*; Wang, Kemin*; Yang, Lijuan; Ding, Jing Acta Chim. Sinica 2012, 70(X), XXX-XXX

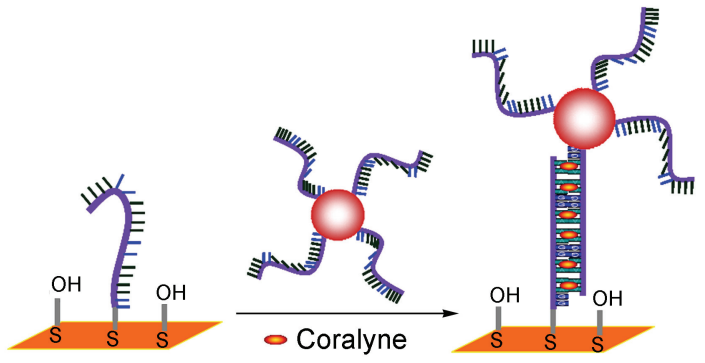

A high sensitive approach for the detection of anticancer drug coralyne based on surface plasmon resonance (SPR) was established. The results showed that the SPR sensor was highly sensitive, and the limit of detection was $c a .0 .07 \mathrm{pmol} / \mathrm{L}$. It was about 5-6 orders of magnitude lower than those of fluorescent and colorimetric methods. 